\title{
An Interplay between Gabor and Wilson Frames
}

\author{
S. K. Kaushik ${ }^{1}$ and Suman Panwar ${ }^{2}$ \\ ${ }^{1}$ Department of Mathematics, Kirori Mal College, University of Delhi, Delhi 110 007, India \\ ${ }^{2}$ Department of Mathematics, University of Delhi, Delhi 110 007, India
}

Correspondence should be addressed to S. K. Kaushik; shikk2003@yahoo.co.in

Received 6 May 2013; Accepted 12 September 2013

Academic Editor: Wilfredo Urbina

Copyright (C) 2013 S. K. Kaushik and S. Panwar. This is an open access article distributed under the Creative Commons Attribution License, which permits unrestricted use, distribution, and reproduction in any medium, provided the original work is properly cited.

Wilson frames $\left\{\psi_{j}^{k}: w_{0}, w_{-1} \in L^{2}(\mathbb{R})\right\}_{j \in \mathbb{Z}, k \in \mathbb{N}_{0}}$ as a generalization of Wilson bases have been defined and studied. We give necessary condition for a Wilson system to be a Wilson frame. Also, sufficient conditions for a Wilson system to be a Wilson Bessel sequence are obtained. Under the assumption that the window functions $w_{0}$ and $w_{-1}$ for odd and even indices of $j$ are the same, we obtain sufficient conditions for a Wilson system to be a Wilson frame (Wilson Bessel sequence). Finally, under the same conditions, a characterization of Wilson frame in terms of Zak transform is given.

\section{Introduction}

In 1946, Gabor [1] proposed a decomposition of a signal in terms of elementary signals, which displays simultaneously the local time and frequency content of the signal, as opposed to the classical Fourier transform which displays only the global frequency content for the entire signal. On the basis of this development, in 1952, Duffin and Schaeffer [2] introduced frames for Hilbert spaces to study some deep problems in nonharmonic Fourier series. In fact, they abstracted the fundamental notion of Gabor for studying signal processing. Janssen [3] showed that while being complete in $L^{2}(\mathbb{R})$, the set suggested by Gabor is not a Riesz basis. This apparent failure of Gabor system was then rectified by resorting to the concept of frames. Since then, the theory of Gabor systems has been intimately related to the theory of frames, and many problems in frame theory find their origins in Gabor analysis. For example, the localized frames were first considered in the realm of Gabor frames [4-7]. Gabor frames have found wide applications in signal and image processing. In view of Balian-Low theorem [8], Gabor frame for $L^{2}(\mathbb{R})$ (which is a Riesz basis) has bad localization properties in time or frequency. Thus, a system to replace Gabor systems which does not have bad localization properties in time and frequency was required. For more literature on Gabor frames one may refer to [8-12]. Wilson et al. [13, 14] suggested a system of functions which are localized around the positive and negative frequency of the same order. The idea of Wilson was used by Daubechies et al. [15] to construct orthonormal "Wilson bases" which consist of functions given by

$$
\begin{gathered}
\psi_{j}^{k}(x)=\left\{\begin{array}{lc}
\varepsilon_{k} \cos (2 k \pi x) w\left(x-\frac{j}{2}\right), & \text { if } j \text { is even, } \\
2 \sin (2(k+1) \pi x) w\left(x-\frac{j+1}{2}\right), & \text { if } j \text { is odd, }
\end{array}\right. \\
\varepsilon_{k}= \begin{cases}\sqrt{2}, & \text { if } k=0, \\
2, & \text { if } k \in \mathbb{N},\end{cases}
\end{gathered}
$$

with a smooth well-localized window function $w$. For such bases the disadvantage described in the Balian-Low theorem is completely removed.

Independently from the work of Daubechies, Jaffard, and Journe, orthonormal local trigonometric bases consisting of the functions $w_{j} \cos (k+(1 / 2)) \pi(\cdot-j), j \in \mathbb{Z}, k \in \mathbb{N}_{0}$ were introduced by Malvar [16]. Some generalizations of Malvar bases exist in [17, 18]. A drawback of Malvar's construction is the restriction on the support of the window functions. But the restriction on orthonormal bases allows only a small class of window functions. In [19], it has been proved that Wilson bases of exponential decay are not unconditional bases for all modulation spaces on $\mathbb{R}$ including the classical Bessel potential space and the Schwartz spaces. Also, Wilson bases 
are not unconditional bases for the ordinary $L^{p}$ spaces for $p \neq 2$, shown in [19]. Approximation properties of Wilson bases are studied in [20]. Wilson bases for general timefrequency lattices are studied in [21]. Generalizations of Wilson bases to nonrectangular lattices are discussed in [13] with motivation from wireless communication and cosines modulated filter banks. Modified Wilson bases are studied in [22]. Bittner [23] considered the Wilson bases introduced by Daubechies et al. with nonsymmetrical window functions for odd and even indices of $j$.

In this paper, we generalize the concept of Wilson bases and define Wilson frames. We give necessary condition for a Wilson system to be a Wilson frame. Also, sufficient conditions for a Wilson system to be a Wilson Bessel sequence are obtained. Under the assumption that the window functions for odd and even indices of $j$ are the same, we obtain sufficient conditions for a Wilson system to be a Wilson frame (Wilson Bessel sequence). Finally, under the same conditions, a characterization of Wilson frame in terms of Zak transform is given.

\section{Preliminaries}

We assume that the reader is familiar with the theory of Gabor frames, refer $[8,9]$ for further details.

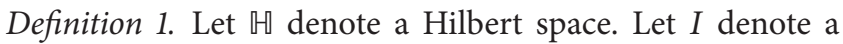
countable index set. A family of vectors $\left\{f_{i}\right\}_{i \in I}$ is called a frame for $\mathbb{H}$ if there exist constants $A$ and $B$ with $0<A \leq$ $B<\infty$ such that

$$
A\|f\|^{2} \leq \sum_{i \in I}\left|\left\langle f, f_{i}\right\rangle\right|^{2} \leq B\|f\|^{2}, \quad \forall f \in \mathbb{M}
$$

The positive constants $A$ and $B$ are called lower frame bound and upper frame bound for the family $\left\{f_{i}\right\}_{i \in I}$, respectively. The inequality (2) is called the frame inequality. If in (2) only the upper inequality holds, then $\left\{f_{i}\right\}_{i \in I}$ is called a Bessel sequence.

Definition 2 (see [9]). Let $g \in L^{2}(\mathbb{R})$ and $a, b$ are positive constants. The sequence $\left\{E_{m b} T_{n a} g\right\}_{m, n \in \mathbb{Z}}$ is called a Gabor system for $L^{2}(\mathbb{R})$. Further,

(i) if $\left\{E_{m b} T_{n a} g\right\}_{m, n \in \mathbb{Z}}$ is a frame for $L^{2}(\mathbb{R})$, then it is called a Gabor frame;

(ii) if $\left\{E_{m b} T_{n a} g\right\}_{m, n \in \mathbb{Z}}$ is a Bessel sequence for $L^{2}(\mathbb{R})$, then it is called a Gabor Bessel sequence.

Definition 3 (see [23]). The Wilson system associated with $w_{0}, w_{-1} \in L^{2}(\mathbb{R})$ is defined as a sequence of functions $\left\{\psi_{j}^{k}\right.$ : $\left.w_{0}, w_{-1} \in L^{2}(\mathbb{R})\right\}_{j \in \mathbb{Z}, k \in \mathbb{N}_{0}}$ in $L^{2}(\mathbb{R})$ given by

$$
\psi_{j}^{k}(x)= \begin{cases}\varepsilon_{k} \cos (2 k \pi x) w_{0}\left(x-\frac{j}{2}\right), & \text { if } j \text { is even, } \\ 2 \sin (2(k+1) \pi x) w_{-1}\left(x-\frac{j+1}{2}\right), & \text { if } j \text { is odd, }\end{cases}
$$

where $\mathbb{N}_{0}=\mathbb{N} \cup\{0\}$ and

$$
\varepsilon_{k}= \begin{cases}\sqrt{2}, & \text { if } k=0 \\ 2, & \text { if } k \in \mathbb{N}\end{cases}
$$

If $w_{0}=w_{-1}=g$, then the Wilson system is given as $\left\{\psi_{j}^{k}:\right.$ $\left.g \in L^{2}(\mathbb{R})\right\}_{j \in \mathbb{Z}, k \in \mathbb{N}_{0}}$.

Definition 4 (see [9]). The Zak transform of $f \in L^{2}(\mathbb{R})$ is defined as a function of two variables given by $(Z f)(t, v)=$ $\sum_{k \in \mathbb{Z}} f(t-k) \exp (2 \pi i k v), t, v \in \mathbb{R}$.

\section{Main Results}

We begin this section with the definition of a Wilson frame.

Definition 5. The Wilson System $\left\{\psi_{j}^{k}: w_{0}, w_{-1} \in L^{2}(\mathbb{R})\right\}_{j \in \mathbb{Z}, k \in \mathbb{N}_{0}}$ for $L^{2}(\mathbb{R})$ associated with $w_{0}, w_{-1} \in L^{2}(\mathbb{R})$ is called a Wilson frame if there exist constants $A$ and $B$ with $0<A \leq B<\infty$ such that

$$
A\|f\|^{2} \leq \sum_{\substack{j \in \mathbb{Z} \\ k \in \mathbb{N}_{0}}}\left|\left\langle f, \psi_{j}^{k}\right\rangle\right|^{2} \leq B\|f\|^{2}, \quad \forall f \in L^{2}(\mathbb{R}) .
$$

The constants $A$ and $B$ are called lower frame bound and upper frame bound, respectively, for the Wilson frame $\left\{\psi_{j}^{k}\right.$ : $\left.w_{0}, w_{-1} \in L^{2}(\mathbb{R})\right\}_{j \in \mathbb{Z}, k \in \mathbb{N}_{0}}$.

Definition 6. In (5), if only the upper inequality holds for all $f \in L^{2}(\mathbb{R})$, then the Wilson system $\left\{\psi_{j}^{k}: w_{0}, w_{-1} \in\right.$ $\left.L^{2}(\mathbb{R})\right\}_{j \in \mathbb{Z}, k \in \mathbb{N}_{0}}$, associated with $w_{0}, w_{-1} \in L^{2}(\mathbb{R})$, is called a Wilson Bessel sequence with Bessel bound $B$.

Example 7. (a) Let $g=w_{0}=w_{-1}=\chi_{[0,1)}$. Then $\left\{\psi_{j}^{k}: g \in\right.$ $\left.L^{2}(\mathbb{R})\right\}_{j \in \mathbb{Z}, k \in \mathbb{N}_{0}}$ is a Wilson frame for $L^{2}(\mathbb{R})$.

(b) Let $w_{0} \neq w_{-1}$ such that $\left|w_{-1}(x)\right| \leq C(1+|x|)^{-1-\epsilon}$, $\left|w_{0}(x)\right| \leq C(1+|x|)^{-1-\epsilon}$ for some constant $C$ and $\epsilon>0$. Let $Q^{+}=(0,1 / 2) \times[-1 / 2,1 / 2]$. Consider the matrix

$$
M(x, \xi)=\left(\frac{\overline{Z w_{0}(x, \xi)}}{-Z w_{-1}(x, \xi)} \frac{\overline{Z w_{0}(-x, \xi)}}{Z w_{-1}(-x, \xi)}\right) .
$$

Let $A_{0}=\operatorname{essinf}_{(x, \xi) \in \mathrm{Q}^{+}}\left\|M^{-1}(x, \xi)\right\|_{2}^{-2}$ and $B_{0}=$ ess $\sup _{(x, \xi) \in \mathrm{Q}^{+}}\|M(x, \xi)\|_{2}^{2}$. If $0<A_{0} \leq B_{0}<\infty$, then the Wilson system $\left\{\psi_{j}^{k}: w_{0}, w_{-1} \in L^{2}(\mathbb{R})\right\}_{j \in \mathbb{Z}, k \in \mathbb{N}_{0}}$ is a Wilson frame for $L^{2}(\mathbb{R})$ with bounds $A_{0}$ and $B_{0}$.

(c) If we choose $g=w_{0}=w_{-1}=\chi_{[0,1 / 2)}$, then $\left\{\psi_{j}^{k}: g \in\right.$ $\left.L^{2}(\mathbb{R})\right\}_{j \in \mathbb{Z}, k \in \mathbb{N}_{0}}$ is not a Wilson frame for $L^{2}(\mathbb{R})$.

(d) Let

$$
w_{0}(x)= \begin{cases}\frac{\sin \pi x}{\pi x}, & \text { if } x \neq 0 \\ 1, & \text { otherwise }\end{cases}
$$


Then $\left\{\psi_{j}^{k}: w_{0} \in L^{2}(\mathbb{R})\right\}_{j \in \mathbb{Z}, k \in \mathbb{N}_{0}}$ is a tight Wilson frame for $L^{2}(\mathbb{R})$ with frame bound 2 .

(e) Let $g(x)=w_{0}(x)=w_{-1}(x)=2^{1 / 2} e^{-x} \chi_{[0, \infty)}(x)$. Then $\left\{\psi_{j}^{k}: g \in L^{2}(\mathbb{R})\right\}_{j \in \mathbb{Z}, k \in \mathbb{N}_{0}}$ is a Wilson frame for $L^{2}(\mathbb{R})$.

(f) Let $g(x)=w_{0}(x)=w_{-1}(x)=2^{1 / 2} /(1+2 \pi i x)$. Then $\left\{\psi_{j}^{k}: g \in L^{2}(\mathbb{R})\right\}_{j \in \mathbb{Z}, k \in \mathbb{N}_{0}}$ is a Wilson frame for $L^{2}(\mathbb{R})$.

(g) If $w_{0}(x)=e^{-\xi(x-1 / 4)^{2}}$ and $w_{-1}(x)=e^{-\xi(x+1 / 4)^{2}}$, where $\xi>0$, then $\left\{\psi_{j}^{k}: w_{0}, w_{-1} \in L^{2}(\mathbb{R})\right\}_{j \in \mathbb{Z}, k \in \mathbb{N}_{0}}$ is a Wilson frame for $L^{2}(\mathbb{R})$.

(h) Let

$$
g(x)= \begin{cases}1+x, & \text { if } x \in[0,1) \\ \frac{x}{2}, & \text { if } x \in[1,2) \\ 0, & \text { otherwise }\end{cases}
$$
$L^{2}(\mathbb{R})$

Then $\left\{\psi_{j}^{k}: g \in L^{2}(\mathbb{R})\right\}_{j \in \mathbb{Z}, k \in \mathbb{N}_{0}}$ is a Wilson frame for

Next, we give two Lemmas which will be used in the subsequent results. Lemma 8 is also proved in [24], but for the sake of completeness, we give the proof.

Lemma 8. Let $\left\{\psi_{j}^{k}: w_{0}, w_{-1} \in L^{2}(\mathbb{R})\right\}_{j \in \mathbb{Z}, k \in \mathbb{N}_{0}}$ be the Wilson system associated with $w_{0}, w_{-1} \in L^{2}(\mathbb{R})$. Then, for $f \in L^{2}(\mathbb{R})$,

$$
\begin{aligned}
\sum_{\substack{j \in \mathbb{Z} \\
k \in \mathbb{N}_{0}}}\left|\left\langle f, \psi_{j}^{k}\right\rangle\right|^{2}=2 \sum_{j, k \in \mathbb{Z}}( & \left|\left\langle f, \cos (2 k \pi \cdot) T_{j} w_{0}(\cdot)\right\rangle\right|^{2} \\
& \left.+\left|\left\langle f, \sin (2 k \pi \cdot) T_{j} w_{-1}(\cdot)\right\rangle\right|^{2}\right) .
\end{aligned}
$$

Proof. Let $f \in L^{2}(\mathbb{R})$. Then

$$
\begin{aligned}
& \sum_{\substack{j \in \mathbb{Z} \\
k \in \mathbb{N}_{0}}}\left|\left\langle f, \psi_{j}^{k}\right\rangle\right|^{2} \\
& =\sum_{\substack{j: \text { even } \\
k \in \mathbb{N}_{0}}}\left|\int f(x) \varepsilon_{k} \cos (2 k \pi x) \overline{w_{0}\left(x-\frac{j}{2}\right)} d x\right|^{2} \\
& \quad+\sum_{\substack{j: \text { odd } \\
k \in \mathbb{N}_{0}}}\left|\int 2 f(x) \sin (2(k+1) \pi x) \overline{w_{-1}\left(x-\frac{j+1}{2}\right)} d x\right|^{2} .
\end{aligned}
$$

This gives

$$
\begin{array}{r}
\sum_{\substack{j \in \mathbb{Z} \\
k \in \mathbb{N}_{0}}}\left|\left\langle f, \psi_{j}^{k}\right\rangle\right|^{2}=2 \sum_{j \in \mathbb{Z}}\left|\int f(x) \overline{w_{0}(x-j)} d x\right|^{2} \\
+4 \sum_{\substack{j \in \mathbb{Z} \\
k \in \mathbb{N}}} \mid \int f(x) \cos (2 k \pi x) \\
\times\left.\overline{w_{0}(x-j)} d x\right|^{2}
\end{array}
$$

$$
\begin{aligned}
+4 \sum_{\substack{j \in \mathbb{Z} \\
k \in \mathbb{N}}} \mid \int f(x) \sin (2 k \pi x) \\
\times\left.\overline{w_{-1}(x-j)} d x\right|^{2} .
\end{aligned}
$$

Thus,

$$
\begin{aligned}
\sum_{\substack{j \in \mathbb{Z} \\
k \in \mathbb{N}_{0}}}\left|\left\langle f, \psi_{j}^{k}\right\rangle\right|^{2}= & 2 \sum_{j \in \mathbb{Z}}\left|\int f(x) \overline{T_{j} w_{0}(x)} d x\right|^{2} \\
& +4 \sum_{\substack{j \in \mathbb{Z} \\
k \in \mathbb{N}}}\left|\left\langle f, \cos (2 k \pi \cdot) T_{j} w_{0}(\cdot)\right\rangle\right|^{2} \\
& +4 \sum_{\substack{j \in \mathbb{Z} \\
k \in \mathbb{N}}}\left|\left\langle f, \sin (2 k \pi \cdot) T_{j} w_{-1}(\cdot)\right\rangle\right|^{2} \\
= & 2 \sum_{j, k \in \mathbb{Z}}\left(\left|\left\langle f, \cos (2 k \pi \cdot) T_{j} w_{0}(\cdot)\right\rangle\right|^{2}\right. \\
& \left.+\left|\left\langle f, \sin (2 k \pi \cdot) T_{j} w_{-1}(\cdot)\right\rangle\right|^{2}\right) .
\end{aligned}
$$

Lemma 9. Let $\left\{\psi_{j}^{k}: w_{0}, w_{-1} \in L^{2}(\mathbb{R})\right\}_{j \in \mathbb{Z}, k \in \mathbb{N}_{0}}$ be the Wilson system associated with $w_{0}, w_{-1} \in L^{2}(\mathbb{R})$. Then, for $f \in L^{2}(\mathbb{R})$,

$$
\begin{aligned}
\sum_{\substack{j \in \mathbb{Z} \\
k \in \mathbb{N}_{0}}}\left|\left\langle f, \psi_{j}^{k}\right\rangle\right|^{2}= & \sum_{j, k \in \mathbb{Z}}\left|\left\langle f, E_{k} T_{j} w_{0}\right\rangle\right|^{2}+\sum_{j, k \in \mathbb{Z}}\left|\left\langle f, E_{k} T_{j} w_{-1}\right\rangle\right|^{2} \\
& +\sum_{j, k \in \mathbb{Z}}\left(\left|\left\langle f, \cos (2 k \pi \cdot) T_{j} w_{0}(\cdot)\right\rangle\right|^{2}\right. \\
& \left.+\left|\left\langle f, \sin (2 k \pi \cdot) T_{j} w_{-1}(\cdot)\right\rangle\right|^{2}\right) \\
& -\sum_{j, k \in \mathbb{Z}}\left(\left|\left\langle f, \cos (2 k \pi \cdot) T_{j} w_{-1}(\cdot)\right\rangle\right|^{2}\right. \\
& \left.+\left|\left\langle f, \sin (2 k \pi \cdot) T_{j} w_{0}(\cdot)\right\rangle\right|^{2}\right) .
\end{aligned}
$$

Proof. We have

$$
\begin{aligned}
\sum_{\substack{j \in \mathbb{Z} \\
k \in \mathbb{N}_{0}}}\left|\left\langle f, \psi_{j}^{k}\right\rangle\right|^{2}= & \sum_{\substack{j: \text { even } \\
k \in \mathbb{N}_{0}}}\left|\left\langle f, \psi_{j}^{k}\right\rangle\right|^{2}+\sum_{\substack{j: \text { odd } \\
k \in \mathbb{N}_{0}}}\left|\left\langle f, \psi_{j}^{k}\right\rangle\right|^{2} \\
= & 2 \sum_{j \in \mathbb{Z}}\left|\left\langle f, T_{j} w_{0}\right\rangle\right|^{2} \\
& +\sum_{\substack{j \in \mathbb{Z} \\
k \in \mathbb{N}}}\left|\left\langle f, E_{k} T_{j} w_{0}+E_{-k} T_{j} w_{0}\right\rangle\right|^{2}
\end{aligned}
$$




$$
\begin{aligned}
& +\sum_{\substack{j \in \mathbb{Z} \\
k \in \mathbb{N}}}\left|\left\langle f, E_{k} T_{j} w_{-1}-E_{-k} T_{j} w_{-1}\right\rangle\right|^{2} \\
= & 2 \sum_{j \in \mathbb{Z}}\left|\left\langle f, T_{j} w_{0}\right\rangle\right|^{2}+\sum_{\substack{j, k \in \mathbb{Z} \\
k \neq 0}}\left|\left\langle f, E_{k} T_{j} w_{0}\right\rangle\right|^{2} \\
+ & \sum_{\substack{j, k \in \mathbb{Z} \\
k \neq 0}}\left|\left\langle f, E_{k} T_{j} w_{-1}\right\rangle\right|^{2} \\
+ & 2 \operatorname{Re} \sum_{\substack{j, k \in \mathbb{Z} \\
k \neq 0}}\left\langle f, E_{k} T_{j} w_{0}\right\rangle \overline{\left\langle f, E_{-k} T_{j} w_{0}\right\rangle} \\
- & \left.2 \operatorname{Re} \sum_{\substack{j, k \in \mathbb{Z} \\
k \neq 0}}\left\langle f, E_{k} T_{j} w_{-1}\right\rangle \overline{\left\langle f, E_{-k} T_{j} w_{-1}\right.}\right\rangle .
\end{aligned}
$$

Therefore, using

$$
\begin{aligned}
\operatorname{Re}\left\langle f, E_{k} T_{j} g\right\rangle \overline{\left\langle f, E_{-k} T_{j} g\right\rangle}= & \left(\left|\left\langle f, \cos (2 k \pi \cdot) T_{j} g(\cdot)\right\rangle\right|^{2}\right. \\
& \left.-\left|\left\langle f, \sin (2 k \pi \cdot) T_{j} g(\cdot)\right\rangle\right|^{2}\right),
\end{aligned}
$$

we finally get the result.

Remark 10. Combining Lemmas 8 and 9, we get

$$
\begin{gathered}
\sum_{j, k \in \mathbb{Z}}\left|\left\langle f, E_{k} T_{j} w_{0}\right\rangle\right|^{2}+\sum_{j, k \in \mathbb{Z}}\left|\left\langle f, E_{k} T_{j} w_{-1}\right\rangle\right|^{2} \\
=\sum_{j, k \in \mathbb{Z}}\left(\left|\left\langle f, \cos (2 k \pi \cdot) T_{j} w_{-1}(\cdot)\right\rangle\right|^{2}\right. \\
\left.+\left|\left\langle f, \sin (2 k \pi \cdot) T_{j} w_{0}(\cdot)\right\rangle\right|^{2}\right) \\
+\sum_{j, k \in \mathbb{Z}}\left(\left|\left\langle f, \cos (2 k \pi \cdot) T_{j} w_{0}(\cdot)\right\rangle\right|^{2}\right. \\
\left.+\left|\left\langle f, \sin (2 k \pi \cdot) T_{j} w_{-1}(\cdot)\right\rangle\right|^{2}\right) .
\end{gathered}
$$

Remark 11. In view of Lemma 8 and Remark 10, the Wilson system obtained by interchanging $w_{0}$ and $w_{-1}$ is also a Wilson Bessel sequence if both the Gabor systems $\left\{E_{k} T_{j} w_{0}\right\}_{k, j \in \mathbb{Z}}$ and $\left\{E_{k} T_{j} w_{-1}\right\}_{k, j \in \mathbb{Z}}$ are Bessel sequences.

The following result gives a necessary condition for a Wilson system $\left\{\psi_{j}^{k}: w_{0}, w_{-1}\right\}_{j \in \mathbb{Z}, k \in \mathbb{N}_{0}}$ associated with $w_{0}, w_{-1} \in$ $L^{2}(\mathbb{R})$ to be a Wilson frame. [9].

The following result is motivated by Proposition 9.1.2 in

Theorem 12. Let $\left\{\psi_{j}^{k}: w_{0}, w_{-1} \in L^{2}(\mathbb{R})_{j \in \mathbb{Z}, k \in \mathbb{N}_{0}}\right.$ be a Wilson frame for $L^{2}(\mathbb{R})$ associated with $w_{0}, w_{-1} \in L^{2}(\mathbb{R})$. Let $A$ denote its lower frame bound. Then

$$
\frac{A}{2} \leq \sum_{j \in \mathbb{Z}}\left(\left|w_{0}(x-j)\right|^{2}+\left|w_{-1}(x-j)\right|^{2}\right) .
$$

More precisely, if the inequality (17) is not satisfied, then the given Wilson system does not satisfy the lower frame condition.

Proof. Assume that condition (17) is violated. Then there exists a measurable set $\Delta \subseteq \mathbb{R}$ having positive measure such that

$$
W(x)=\sum_{j \in \mathbb{Z}}\left(\left|w_{0}(x-j)\right|^{2}+\left|w_{-1}(x-j)\right|^{2}\right)<\frac{A}{2} \quad \text { on } \Delta .
$$

We can assume that this $\Delta$ is contained in an interval of length 1. Let

$$
\begin{gathered}
\Delta_{0}=\left\{x \in \Delta: W(x) \leq \frac{A}{2}-1\right\}, \\
\Delta_{k}=\left\{x \in \Delta: \frac{A}{2}-\frac{1}{k}<W(x)<\frac{A}{2}-\frac{1}{k+1}\right\} .
\end{gathered}
$$

Then $\Delta$ is partitioned into disjoint measurable sets such that at least one of these measurable sets will have a positive measure. Let this set be $\Delta_{k^{\prime}}$. Choose $f=\chi_{\Delta_{k^{\prime}}}$. Then $\|f\|=\left|\chi_{\Delta_{k^{\prime}}}\right|$, where $\left|\chi_{\Delta_{k^{\prime}}}\right|=$ measure of $\chi_{\Delta_{k^{\prime}}}$.

Since for $j \in \mathbb{Z}$, the functions $f \overline{T_{j} w_{0}}$ and $f \overline{T_{j} w_{-1}}$ have support in $\Delta_{k^{\prime}},\left\{E_{k}\right\}_{k \in \mathbb{Z}}$ constitute an orthonormal basis for $L^{2}(I)$, for every interval $I$ of length 1 and $\Delta_{k^{\prime}}$ is contained in an interval of length 1 , we have

$$
\begin{aligned}
\sum_{k \in \mathbb{Z}}\left|\left\langle f, E_{k} T_{j} w_{0}\right\rangle\right|^{2} & =\sum_{k \in \mathbb{Z}}\left|\left\langle f \overline{T_{j} w_{0}}, E_{k}\right\rangle\right|^{2} \\
& =\int_{\mathbb{R}}|f(x)|^{2}\left|w_{0}(x-j)\right|^{2} d x .
\end{aligned}
$$

Also, since $f=\chi_{\Delta_{k^{\prime}}}$, we have

$$
\sum_{j, k \in \mathbb{Z}}\left|\left\langle f, E_{k} T_{j} w_{0}\right\rangle\right|^{2}=\sum_{j \in \mathbb{Z}} \int_{\Delta_{k^{\prime}}}\left|w_{0}(x-j)\right|^{2} d x
$$

Similarly, we obtain

$$
\sum_{j, k \in \mathbb{Z}}\left|\left\langle f, E_{k} T_{j} w_{-1}\right\rangle\right|^{2}=\sum_{j \in \mathbb{Z}} \int_{\Delta_{k^{\prime}}}\left|w_{-1}(x-j)\right|^{2} d x .
$$

Therefore,

$$
\begin{aligned}
\sum_{j, k \in \mathbb{Z}}\left(\left|\left\langle f, E_{k} T_{j} w_{0}\right\rangle\right|^{2}+\left|\left\langle f, E_{k} T_{j} w_{-1}\right\rangle\right|^{2}\right) \\
=\sum_{j \in \mathbb{Z}} \int_{\Delta_{k^{\prime}}}\left(\left|w_{0}(x-j)\right|^{2}+\left|w_{-1}(x-j)\right|^{2}\right) d x \\
=\int_{\Delta_{k^{\prime}}} W(x) d x .
\end{aligned}
$$


Further, since

$$
\begin{aligned}
& \sum_{j \in \mathbb{Z}, k \in \mathbb{N}_{0}}\left|\left\langle f, \psi_{j}^{k}\right\rangle\right|^{2} \\
& \quad \leq 2 \sum_{j, k \in \mathbb{Z}}\left(\left|\left\langle f, E_{k} T_{j} w_{0}\right\rangle\right|^{2}+\left|\left\langle f, E_{k} T_{j} w_{-1}\right\rangle\right|^{2}\right),
\end{aligned}
$$

we get

$$
\begin{aligned}
\sum_{\substack{j \in \mathbb{Z} \\
k \in \mathbb{N}_{0}}}\left|\left\langle f, \psi_{j}^{k}\right\rangle\right|^{2} & \leq 2 \int_{\Delta_{k^{\prime}}} W(x) d x \\
& \leq 2\left(\frac{A}{2}-\frac{1}{k^{\prime}+1}\right) \int_{\Delta_{k^{\prime}}} d x \\
& =\left(A-\frac{2}{k^{\prime}+1}\right)\|f\|^{2} .
\end{aligned}
$$

Hence,

$$
\sum_{j, k \in \mathbb{Z}}\left|\left\langle f, \psi_{j}^{k}\right\rangle\right|^{2}<A\|f\|^{2}
$$

This is a contradiction.

Next, we give a sufficient condition for a Wilson system $\left\{\psi_{j}^{k}: w_{0}, w_{-1} \in L^{2}(\mathbb{R})\right\}_{j \in \mathbb{Z}, k \in \mathbb{N}_{0}}$ to be a Wilson Bessel sequence.

Theorem 13. Let $w_{0}, w_{-1} \in L^{2}(\mathbb{R})$,

$$
\begin{gathered}
B_{1}=\sup _{x \in[0,1]} \sum_{k \in \mathbb{Z}}\left|\sum_{n \in \mathbb{Z}} w_{0}(x-n) \overline{w_{0}(x-n-k)}\right|<\infty, \\
B_{2}=\sup _{x \in[0,1]} \sum_{k \in \mathbb{Z}}\left|\sum_{n \in \mathbb{Z}} w_{-1}(x-n) \overline{w_{-1}(x-n-k)}\right|<\infty .
\end{gathered}
$$

Then, the Wilson system $\left\{\psi_{j}^{k}: w_{0}, w_{-1} \in L^{2}(\mathbb{R})\right\}_{j \in \mathbb{Z}, k \in \mathbb{N}_{0}}$ is a Wilson Bessel sequence with Bessel bound $2\left(B_{1}+B_{2}\right)$.

Proof. In view of Theorem 9.1.5 in [9], the Gabor systems $\left\{E_{k} T_{j} w_{0}\right\}_{k, j \in \mathbb{Z}}$ and $\left\{E_{k} T_{j} w_{-1}\right\}_{k, j \in \mathbb{Z}}$ are Gabor Bessel sequences. Therefore, using Lemma 8 and Remark 10, the Wilson system $\left\{\psi_{j}^{k}: w_{0}, w_{-1} \in L^{2}(\mathbb{R})\right\}_{j \in \mathbb{Z}, k \in \mathbb{N}_{0}}$ is a Wilson Bessel sequence with Bessel bound $2\left(B_{1}+B_{2}\right)$.

Corollary 14. Let $w_{0}, w_{-1} \in L^{2}(\mathbb{R})$ be bounded and compactly supported. Then the Wilson system $\left\{\psi_{j}^{k}: w_{0}, w_{-1} \in\right.$ $\left.L^{2}(\mathbb{R})\right\}_{j \in \mathbb{Z}, k \in \mathbb{N}_{0}}$ is a Wilson Bessel sequence.

Proof. Since, $w_{0}, w_{-1} \in L^{2}(\mathbb{R})$ are bounded and compactly supported, $B_{1}$ and $B_{2}$ as defined in Theorem 13 are both finite, and hence, the Wilson system $\left\{\psi_{j}^{k}: w_{0}, w_{-1} \in L^{2}(\mathbb{R})\right\}_{j \in \mathbb{Z}, k \in \mathbb{N}_{0}}$ is a Wilson Bessel sequence.

In the following results, we give a sufficient condition for the Wilson system $\left\{\psi_{j}^{k}: w_{0}, w_{-1} \in L^{2}(\mathbb{R})\right\}_{j \in \mathbb{Z}, k \in \mathbb{N}_{0}}$ to be a Wilson Bessel sequence in terms of Zak transforms of $w_{0}$ and $w_{-1}$.
Theorem 15. Let $w_{0}, w_{-1} \in L^{2}(\mathbb{R})$, and let there exist $B_{1}>$ $0, B_{2}>0$ such that $\left|Z w_{0}\right|^{2} \leq B_{1}$ and $\left|Z w_{-1}\right|^{2} \leq B_{2}$. Then, the Wilson system $\left\{\psi_{j}^{k}: w_{0}, w_{-1} \in L^{2}(\mathbb{R})\right\}_{j \in \mathbb{Z}, k \in \mathbb{N}_{0}}$ is a Wilson Bessel sequence with Bessel bound $2\left(B_{1}+B_{2}\right)$.

Proof. In view of Proposition 9.7.3 in [9], the Gabor systems $\left\{E_{k} T_{j} w_{0}\right\}_{k, j \in \mathbb{Z}}$ and $\left\{E_{k} T_{j} w_{-1}\right\}_{k, j \in \mathbb{Z}}$ are Gabor Bessel sequences with Bessel bounds $B_{1}$ and $B_{2}$, respectively. Therefore, using Lemma 8 and Remark 10, the Wilson system $\left\{\psi_{j}^{k}: w_{0}, w_{-1} \in\right.$ $\left.L^{2}(\mathbb{R})\right\}_{j \in \mathbb{Z}, k \in \mathbb{N}_{0}}$ is a Wilson Bessel sequence with Bessel bound $2\left(B_{1}+B_{2}\right)$.

Next, we give sufficient conditions for a Wilson system $\left\{\psi_{j}^{k}: w_{0} \in L^{2}(\mathbb{R})\right\}_{j \in \mathbb{Z}, k \in \mathbb{N}_{0}}$ to be a Wilson frame.

Theorem 16. Let $w_{0} \in L^{2}(\mathbb{R})$ and

$$
B=\sup _{x \in[0,1]} \sum_{k \in \mathbb{Z}}\left|\sum_{n \in \mathbb{Z}} w_{0}(x-n) \overline{w_{0}(x-n-k)}\right|<\infty .
$$

Then the Wilson system $\left\{\psi_{j}^{k}: w_{0} \in L^{2}(\mathbb{R}\}_{j \in \mathbb{Z}, k \in \mathbb{Z}_{0}}\right.$ is a Wilson Bessel sequence. Further, if

$$
\begin{aligned}
A=\inf _{x \in[0,1]}[ & \sum_{n \in \mathbb{Z}}\left|w_{0}(x-n)\right|^{2} \\
& \left.-\sum_{k \neq 0}\left|\sum_{n \in \mathbb{Z}} w_{0}(x-n) \overline{w_{0}(x-n-k)}\right|\right]>0,
\end{aligned}
$$

then the Wilson system $\left\{\psi_{j}^{k}: w_{0} \in L^{2}(\mathbb{R})\right\}_{j \in \mathbb{Z}, k \in \mathbb{N}_{0}}$ is a Wilson frame with frame bounds $A / 2$ and $B / 2$.

Proof. In view of Theorem 9.1.5 in [9], the Gabor system $\left\{E_{k} T_{j} w_{0}\right\}_{k, j \in \mathbb{Z}}$ is a Gabor frame for $L^{2}(\mathbb{R})$. If we choose $w_{0}=$ $w_{-1}$ in Lemma 9, then

$$
\sum_{\substack{j \in \mathbb{Z} \\ k \in \mathbb{N}_{0}}}\left|\left\langle f, \psi_{j}^{k}\right\rangle\right|^{2}=2 \sum_{j, k \in \mathbb{Z}}\left|\left\langle f, E_{k} T_{j} w_{0}\right\rangle\right|^{2} \quad \forall f \in L^{2}(\mathbb{R}) .
$$

Hence, the Wilson system $\left\{\psi_{j}^{k}: w_{0} \in L^{2}(\mathbb{R})\right\}_{j \in \mathbb{Z}, k \in \mathbb{N}_{0}}$ is a Wilson frame with frame bounds $A / 2$ and $B / 2$.

Corollary 17. Suppose $w_{0} \in L^{2}(\mathbb{R})$ has support in an interval of length 1 ; then the Wilson system $\left\{\psi_{j}^{k}: w_{0} \in L^{2}(\mathbb{R})\right\}_{j \in \mathbb{Z}, k \in \mathbb{N}_{0}}$ is a Wilson frame for $L^{2}(\mathbb{R})$.

Proof. Since $w_{0} \in L^{2}(\mathbb{R})$ has support in an interval of length 1 , we have $\sum_{n \in \mathbb{Z}} w_{0}(x-n) \overline{w_{0}(x-n-k)}=0$, for all $k \neq 0$. Thus, $B<\infty, A>0$. Hence, in view of Theorem 16, the Wilson system $\left\{\psi_{j}^{k}: w_{0} \in L^{2}(\mathbb{R})\right\}_{j \in \mathbb{Z}, k \in \mathbb{N}_{0}}$ is a frame for $L^{2}(\mathbb{R})$.

The following result gives a class of functions $w_{0} \in L^{2}(\mathbb{R})$ for which the associated Wilson system is a Bessel sequence but not a frame. 
Theorem 18. Suppose $w_{0} \in L^{2}(\mathbb{R})$ is a continuous function with compact support. Then the Wilson system $\left\{\psi_{j}^{k}: w_{0} \in\right.$ $\left.L^{2}(\mathbb{R})\right\}_{j \in \mathbb{Z}, k \in \mathbb{N}_{0}}$ is a Wilson Bessel sequence for $L^{2}(\mathbb{R})$ but not a frame.

Proof. Since $w_{0} \in L^{2}(\mathbb{R})$ is a bounded function with compact support, $B$ defined in Theorem 16 is finite, and hence, the Wilson system $\left\{\psi_{j}^{k}: w_{0} \in L^{2}(\mathbb{R}\}_{j \in \mathbb{Z}, k \in \mathbb{N}_{0}}\right.$ is a Wilson Bessel sequence for $L^{2}(\mathbb{R})$. Moreover, since $w_{0} \in L^{2}(\mathbb{R})$ is a continuous function with compact support, in view of corollary 9.7.4 in [9], the Gabor system $\left\{E_{k} T_{j} w_{0}\right\}_{k, j \in \mathbb{Z}}$ can never become a frame, and hence, the Wilson system $\left\{\psi_{j}^{k}\right.$ : $w_{0} \in L^{2}(\mathbb{R}\}_{j \in \mathbb{Z}, k \in \mathbb{N}_{0}}$ is not a Wilson frame.

Finally, we give a necessary and sufficient condition for a Wilson system $\left\{\psi_{j}^{k}: w_{0} \in L^{2}(\mathbb{R}\}_{j \in \mathbb{Z}, k \in \mathbb{N}_{0}}\right.$ to be a Wilson frame in terms of the Zak transform of $w_{0}$.

Theorem 19. The Wilson system $\left\{\psi_{j}^{k}: w_{0} \in L^{2}(\mathbb{R}\}_{j \in \mathbb{Z}, k \in \mathbb{N}_{0}}\right.$ is a Wilson frame for $L^{2}(\mathbb{R})$ with bounds $2 A$ and $2 B$ if and only if $A \leq\left|Z w_{0}\right|^{2} \leq B$.

Proof. If we choose $w_{0}=w_{-1}$ in Lemma 9, then

$$
\sum_{\substack{j \in \mathbb{Z} \\ k \in \mathbb{N}_{0}}}\left|\left\langle f, \psi_{j}^{k}\right\rangle\right|^{2}=2 \sum_{j, k \in \mathbb{Z}}\left|\left\langle f, E_{k} T_{j} w_{0}\right\rangle\right|^{2} \quad \forall f \in L^{2}(\mathbb{R}) .
$$

Therefore, the Wilson system $\left\{\psi_{j}^{k}: w_{0} \in L^{2}(\mathbb{R}\}_{j \in \mathbb{Z}, k \in \mathbb{N}_{0}}\right.$ is a Wilson frame for $L^{2}(\mathbb{R})$ with bounds $2 A$ and $2 B$ if and only if the Gabor system $\left\{E_{k} T_{j} w_{0}\right\}_{k, j \in \mathbb{Z}}$ is a frame for $L^{2}(\mathbb{R})$ with bounds $A$ and $B$. Since, the Gabor system $\left\{E_{k} T_{j} w_{0}\right\}_{k, j \in \mathbb{Z}}$ is a frame for $L^{2}(\mathbb{R})$ with bounds $A$ and $B$ if and only if $A \leq\left|Z w_{0}\right|^{2} \leq B$, the result follows.

\section{Acknowledgments}

The authors thank the referee(s) for their useful suggestions and comments towards the improvement of the paper. The research of Suman Panwar is supported by CSIR vide letter no. $09 \backslash 045(1140) \backslash 2011-E M R$ I dated $16 \backslash 11 \backslash 2011$.

\section{References}

[1] D. Gabor, "Theory of communications," Journal of the Institution of Electrical Engineers, vol. 93, no. 3, pp. 429-457, 1946.

[2] R. J. Duffin and A. C. Schaeffer, "A class of nonharmonic Fourier series," Transactions of the American Mathematical Society, vol. 72, no. 2, pp. 341-366, 1952.

[3] A. J. E. M. Janssen, "Gabor representation of generalized functions," Journal of Mathematical Analysis and Applications, vol. 83, no. 2, pp. 377-394, 1981.

[4] R. Balan, P. G. Casazza, C. Heil, and Z. Landau, "Density, overcompleteness, and localization of frames. I. Theory," The Journal of Fourier Analysis and Applications, vol. 12, no. 2, pp. 105-143, 2006.
[5] R. Balan, P. G. Casazza, C. Heil, and Z. Landau, "Density, overcompleteness, and localization of frames. II. Gabor systems," The Journal of Fourier Analysis and Applications, vol. 12, no. 3, pp. 309-344, 2006.

[6] P. G. Casazza and G. E. Pfander, "Infinite dimensional restricted invertibility," Journal of Functional Analysis, vol. 263, no. 12, pp. 3784-3803, 2012.

[7] K. Gröchenig, "Localization of frames, Banach frames, and the invertibility of the frame operator," The Journal of Fourier Analysis and Applications, vol. 10, no. 2, pp. 105-132, 2004.

[8] K. Gröchenig, Foundations of Time-Frequency Analysis, Applied and Numerical Harmonic Analysis, Birkhäuser, Boston, Mass, USA, 2001.

[9] O. Christensen, Frames and Bases: An Introductory Course, Applied and Numerical Harmonic Analysis, Birkhäuser, Boston, Mass, USA, 2008.

[10] H. G. Feichtinger and T. Strohmer, Eds., Gabor Analysis and Algorithms: Theory and Applications, Applied and Numerical Harmonic Analysis, Birkhäuser, Boston, Mass, USA, 1998.

[11] H. G. Feichtinger and T. Strohmer, Eds., Advances in Gabor Analysis, Applied and Numerical Harmonic Analysis, Birkhäuser, Boston, Mass, USA, 2003.

[12] T. Strohmer, "Approximation of dual Gabor frames, window decay, and wireless communications," Applied and Computational Harmonic Analysis, vol. 11, no. 2, pp. 243-262, 2001.

[13] D. J. Sullivan, J. J. Rehr, J. W. Wilkins, and K. G. Wilson, Phase Space Warnier Functions in Electronic Structure Calculations, Cornell University, 1987.

[14] K. G. Wilson, Generalised Warnier Functions, Cornell university, 1987.

[15] I. Daubechies, S. Jaffard, and J.-L. Journé, "A simple Wilson orthonormal basis with exponential decay," SIAM Journal on Mathematical Analysis, vol. 22, no. 2, pp. 554-573, 1991.

[16] H. S. Malvar, "Lapped transforms for efficient transform/subband coding," IEEE Transactions on Acoustics, Speech, and Signal Processing, vol. 38, no. 6, pp. 969-978, 1990.

[17] P. Auscher, G. Weiss, and M. V. Wickerhauser, "Local sine and cosine bases of Coifman and Meyer and the construction of smooth wavelets," in Wavelets: A Tutorial in Theory and Applications, C. K. Chui, Ed., vol. 2 of Wavelet Analysis and Its Applications, pp. 237-256, Academic Press, Boston, Mass, USA, 1992.

[18] R. R. Coifman and Y. Meyer, "Remarques sur l'analyse de Fourier à fenêtre," Comptes Rendus de l'Académie des Sciences, vol. 312, no. 3, pp. 259-261, 1991.

[19] H. G. Feichtinger, K. Gröchenig, and D. Walnut, "Wilson bases and modulation spaces," Mathematische Nachrichten, vol. 155, pp. 7-17, 1992.

[20] K. Bittner, "Linear approximation and reproduction of polynomials by Wilson bases," The Journal of Fourier Analysis and Applications, vol. 8, no. 1, pp. 85-108, 2002.

[21] G. Kutyniok and T. Strohmer, "Wilson bases for general timefrequency lattices," SIAM Journal on Mathematical Analysis, vol. 37, no. 3, pp. 685-711, 2005.

[22] P. Wojdyłło, "Modified Wilson orthonormal bases," Sampling Theory in Signal and Image Processing, vol. 6, no. 2, pp. 223-235, 2007.

[23] K. Bittner, "Biorthogonal Wilson bases," in Wavelet Applications in Signal and Image Processing VII, vol. 3813 of Proceedings of SPIE, pp. 410-421, Denver, Colo, USA, July 1999.

[24] S. K. Kaushik and S. Panwar, "A note on Gabor frames," Communicated. 


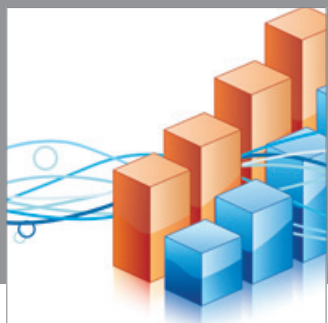

Advances in

Operations Research

mansans

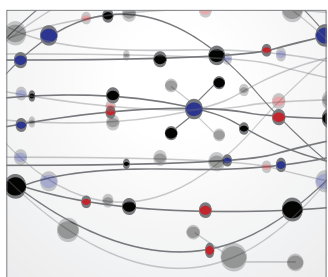

The Scientific World Journal
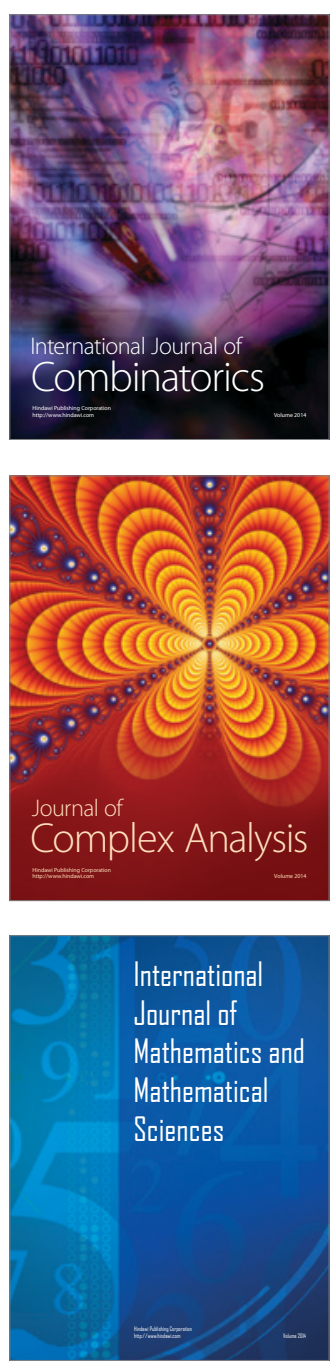
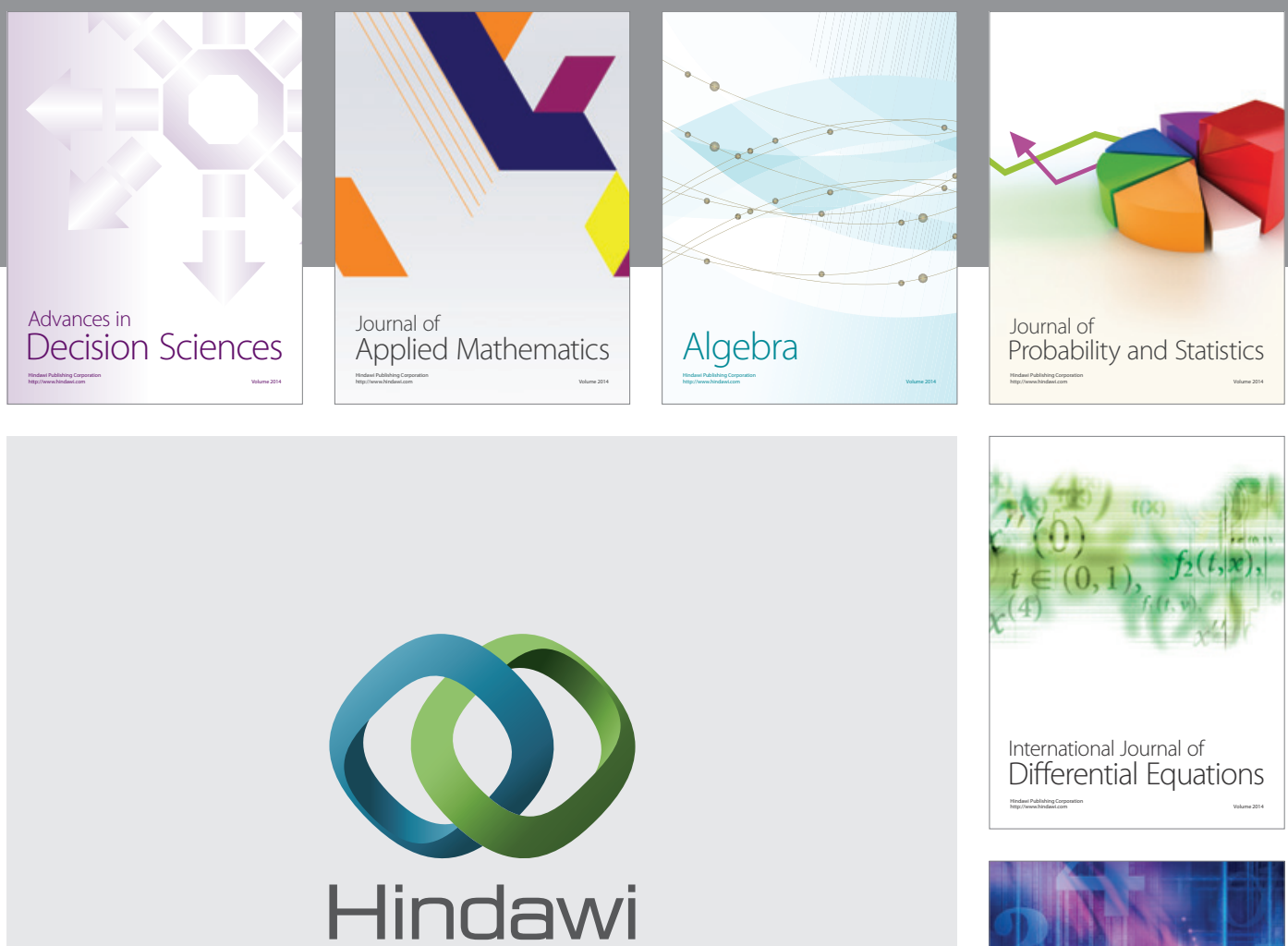

Submit your manuscripts at http://www.hindawi.com
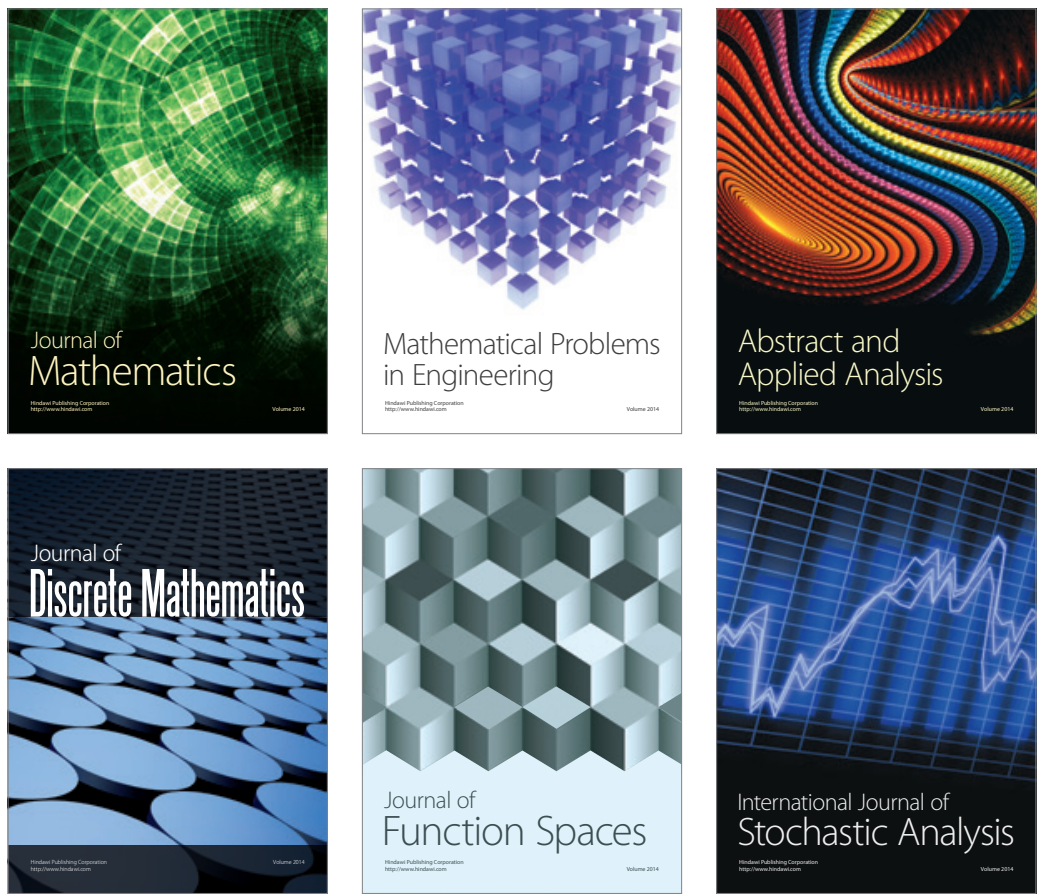

Journal of

Function Spaces

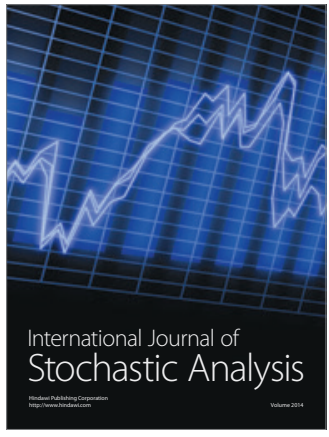

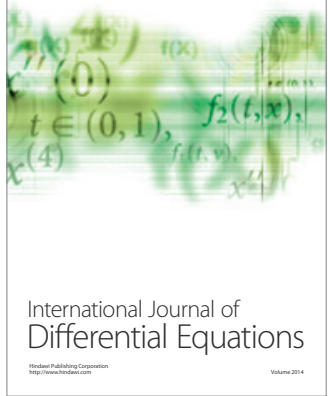
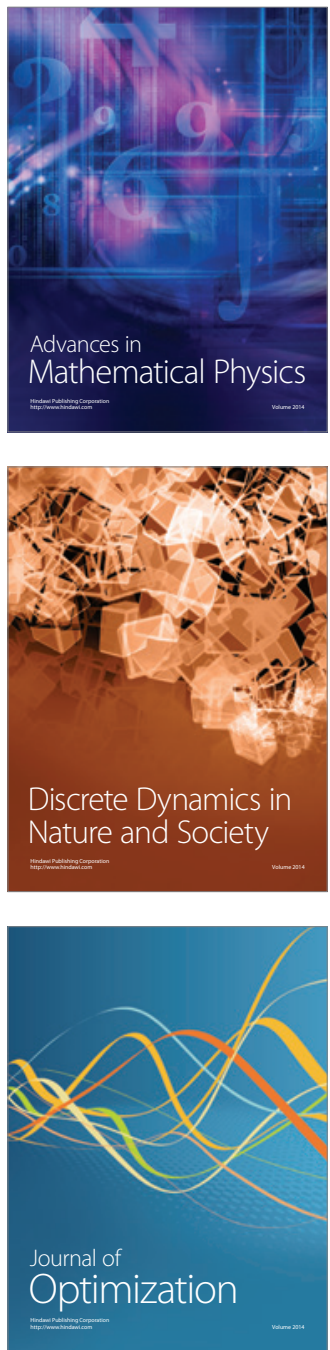